تأثير شكل قصبة المحراث الحفار في متطلبات القدرة للساحبة

محمود الياس احمد الطائي

$$
\begin{aligned}
& \text { سعد عبد الجبار الرجبو } \\
& \text { قسم المكنتة الزراعية/ كلية الزراعة والغابات } \\
& \text { قسم المكائن والمعدات الزية }
\end{aligned}
$$

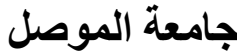

$$
\begin{aligned}
& \text { المعهل التقني/الموصز }
\end{aligned}
$$

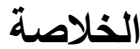

أجريت هذه الدر اسة في الموسم (2005 - 2006) بمحافظة نينوى وفي موقعين مختلفي

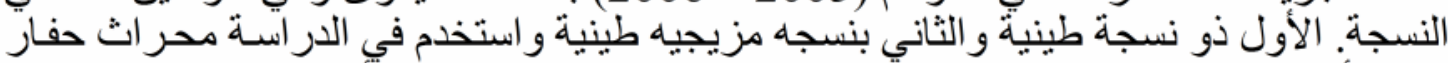

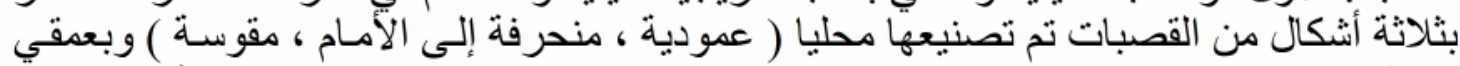
‘)(12-8)

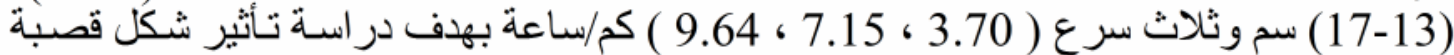

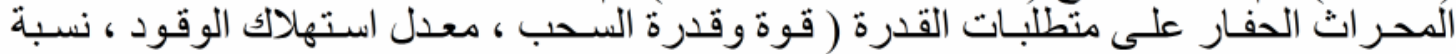

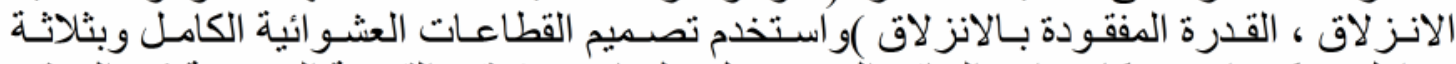

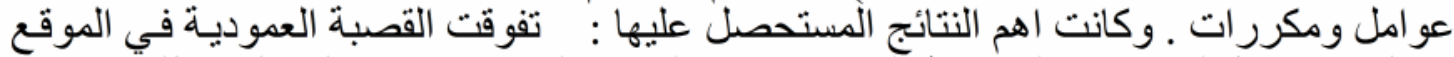

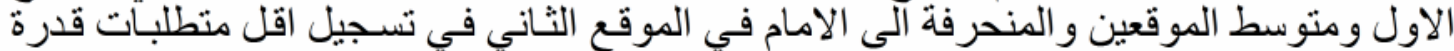

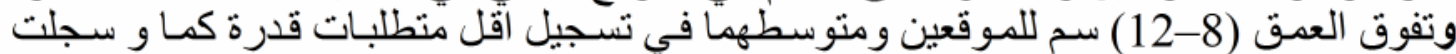

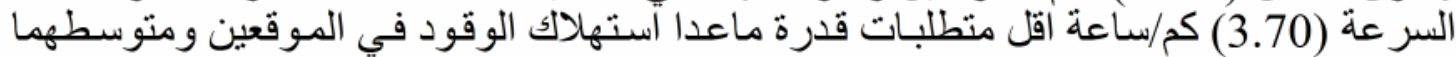

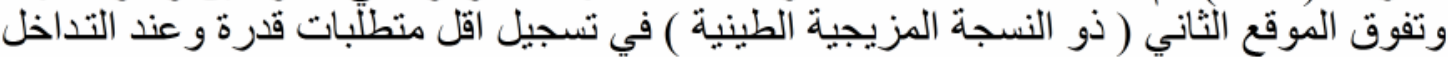

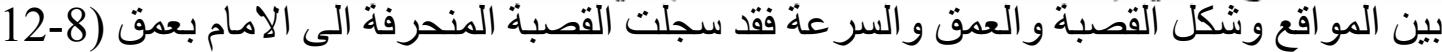
) سم وسرعة (3.70 ) كم/ساعة في الموقع الثاني اقل منطلبات قدرة ما عدا استهلاك الوقد الكود.

\title{
Effect of chisel plow shanks shape on power \\ Requirements of Tractor
}

Saad Abdul Jabbar Al- Rajaboo

Mahmood Elias Ahmed Al-

Taee

College of Agriculture \&Forestry

Technical Institute of Mosul

University of Mosul 


\begin{abstract}
This study was carried out at two sites of different soil texture, clay and a clay loam during the season of 2005 - 2006 In Mosul .A plough of three different shank shapes have been used. These shapes were vertical, inclined forward and curved with two plough depths of 8-12 cms and 13$17 \mathrm{cms}$ successively with three speeds $3.70,7.15$ and $9.64 \mathrm{~km} /$ hour. In order to study the effect of the shank shape on the power requirements which include the draft force, rate of fuel consumption, slippage ratio and the power loss due to slippage. The two experimental farms have been designed by the use of (RCBD) with three factors and replications. The main results of this study, The vertical shank outperformed at the first site and the inclined forward outperformed at the second site in recording the lowest requirement with their means, the $8-12 \mathrm{~cm}$ depth outperformed at the two sites with their means in recording the lowest power requirement, the $(3.70) \mathrm{km} /$ hour speed recorded the lowest power requirement except fuel consumption at the two sites with their means, The second site (clay loam texture) outperformed records the lowest power requirements.and at the effect of interaction between the sites, shank shape, depth and speed on the studied properties the inclined forward shank at depth of $(8-12) \mathrm{cm}$ and at speed of (3.70) $\mathrm{km} /$ hour gave the lowest power requirement at the second site except fuel consumption.
\end{abstract}

Keyword: Tillage - Chisel Plow Shanks - Power requirement 
هناك آنواع مختلفة من المحاريث الحفارة المستخدمة في العالم على اساس نوع الاسلحة

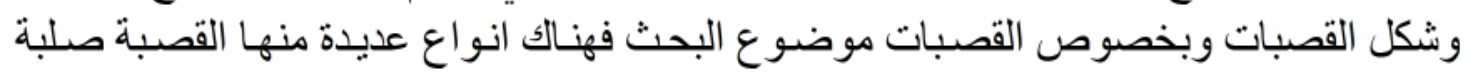
القو ائم ومرنة القو ائم من خلال نابض تسمى القصبات ذات النو ابض. أو مرنـة من خـلاتل بنائهـا

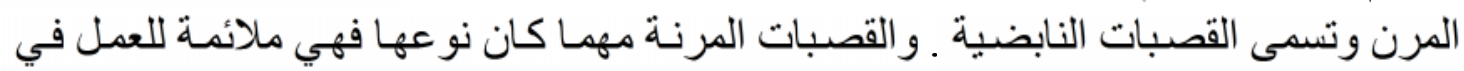

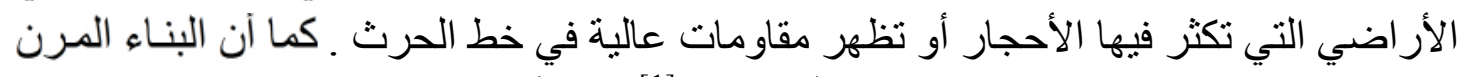

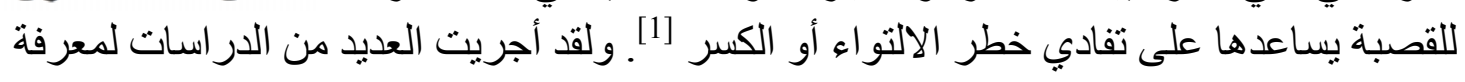

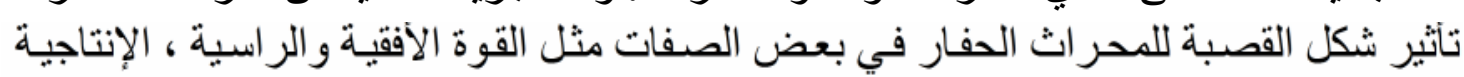

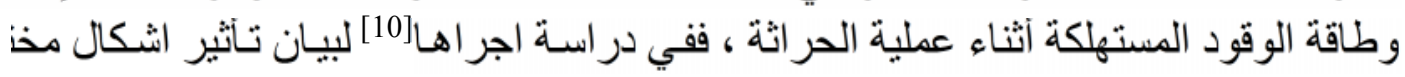

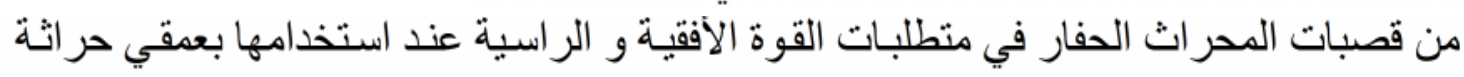

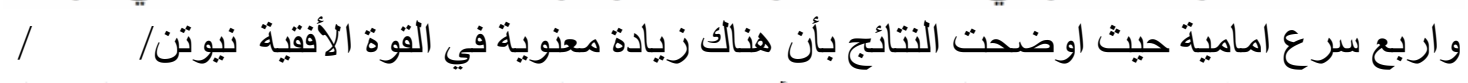

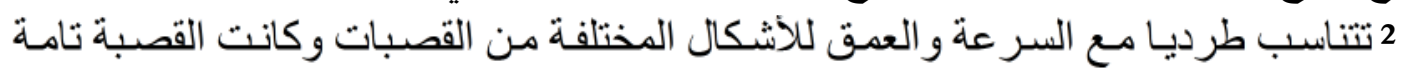

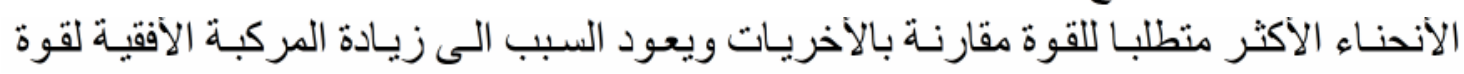

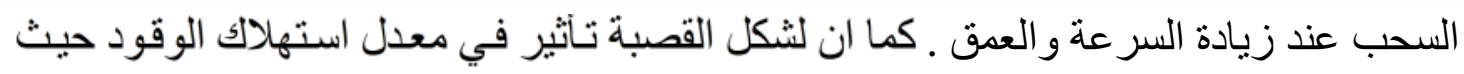

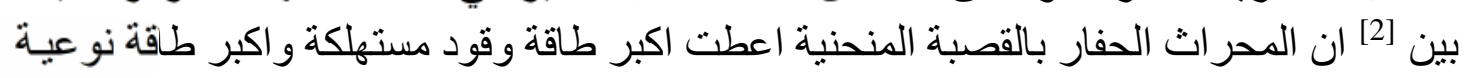

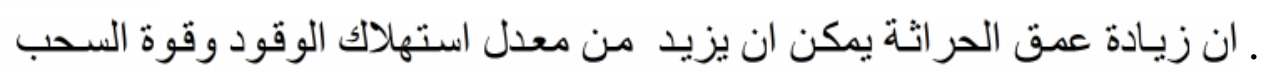

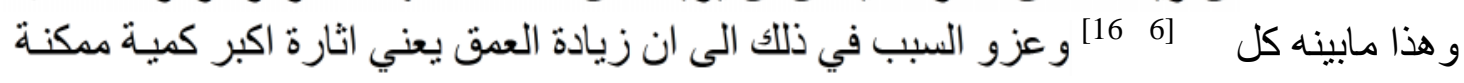

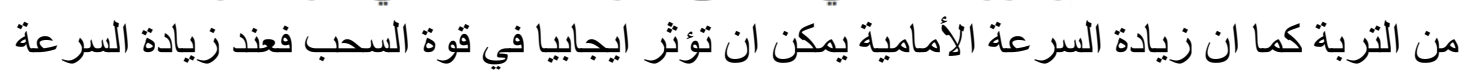

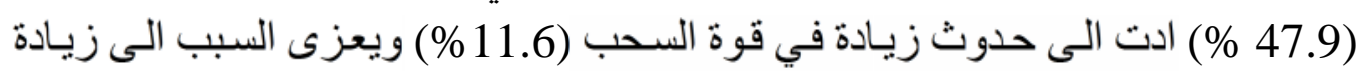

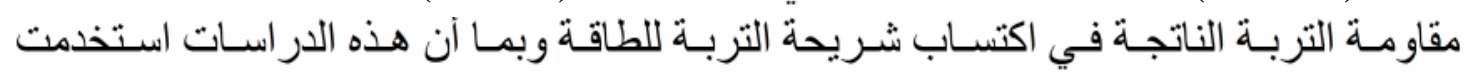

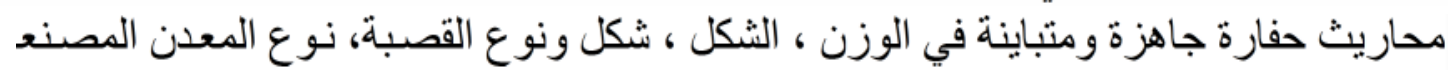

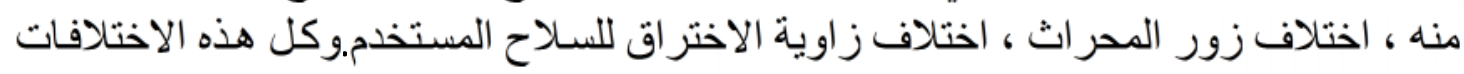

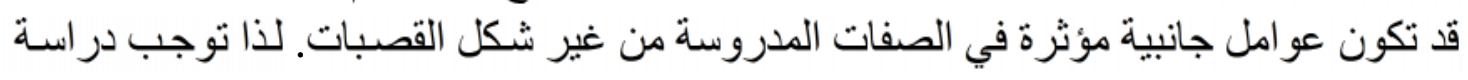

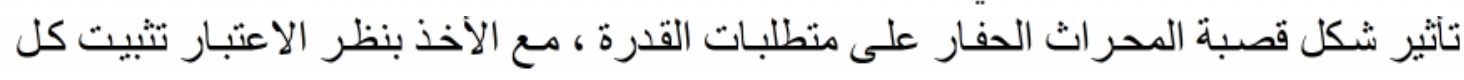

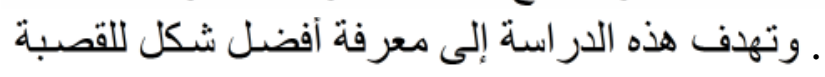

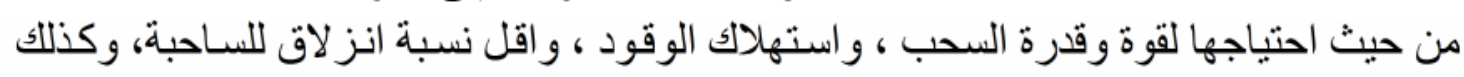

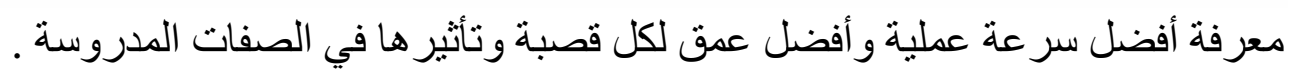

\section{مواد البحث وطرائقه}

تم تحديـد مـوقعين في منطقة الرشـيدية بمحافظـة نينـوى مختلفي النسـجة ( طينيـة ) (مزيجيـة طينيـة ) وكانت مسـاحة كل موقع ( 5400 ) 2 م

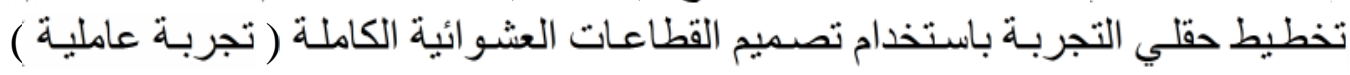

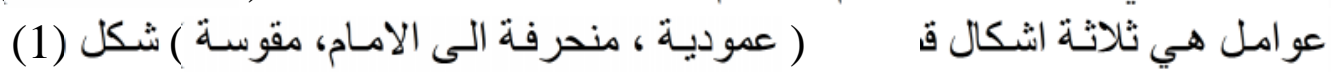

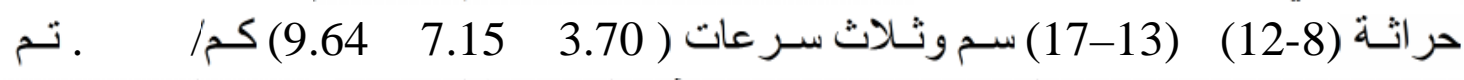
تصنيع هذه القصبات صلبة القوائم في المعامل الأهلية بمحافظة نينوى ( منحرفة الى الامـام 
) بعد الاتفاق على المعدن الذي تم تصنيع

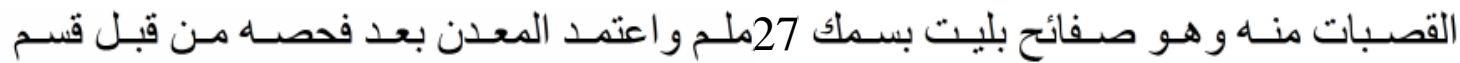

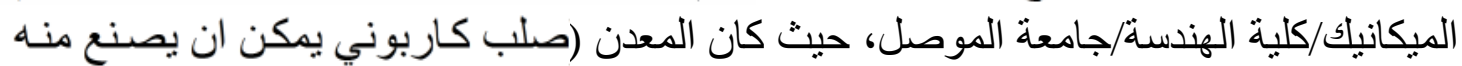

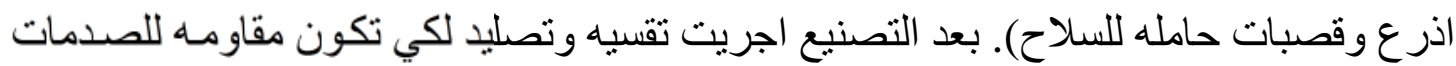

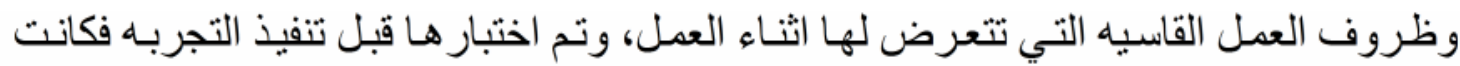

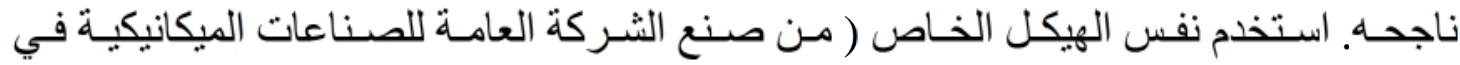

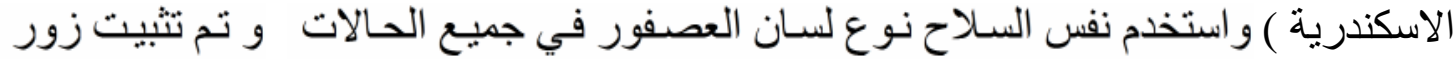

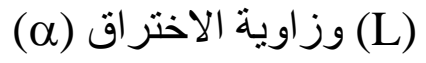

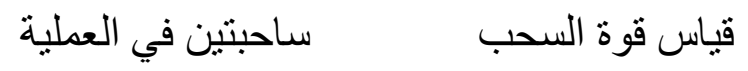

سلسلة ووضع بينهما المقياس ( الداينموميتر ) ووتم حساب قوة السحب للمحر اث وكما يلي : -

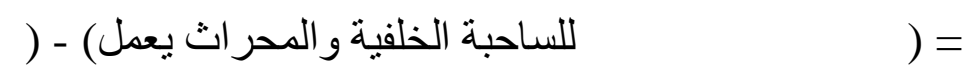

المطلوبة لسحب الساحبة الخلفية والمحر اث معلق خلفها بدون عمل )

$$
\text { × أما قدرة السحب فهي قدرة الساحبة المقاسة على قضيب الجر وهي حاصل ضرب ( }
$$

$$
\mathrm{Dp}=\mathrm{Pk} \times \mathrm{V} \times 0.746 / 270
$$

$$
\begin{aligned}
& \text { ( } \quad \text { (كيلوواط } \quad \text { ( } \\
& (\mathrm{kg}) \quad=\mathrm{Pk}
\end{aligned}
$$

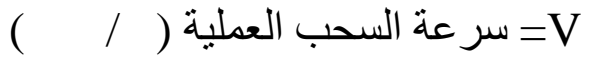
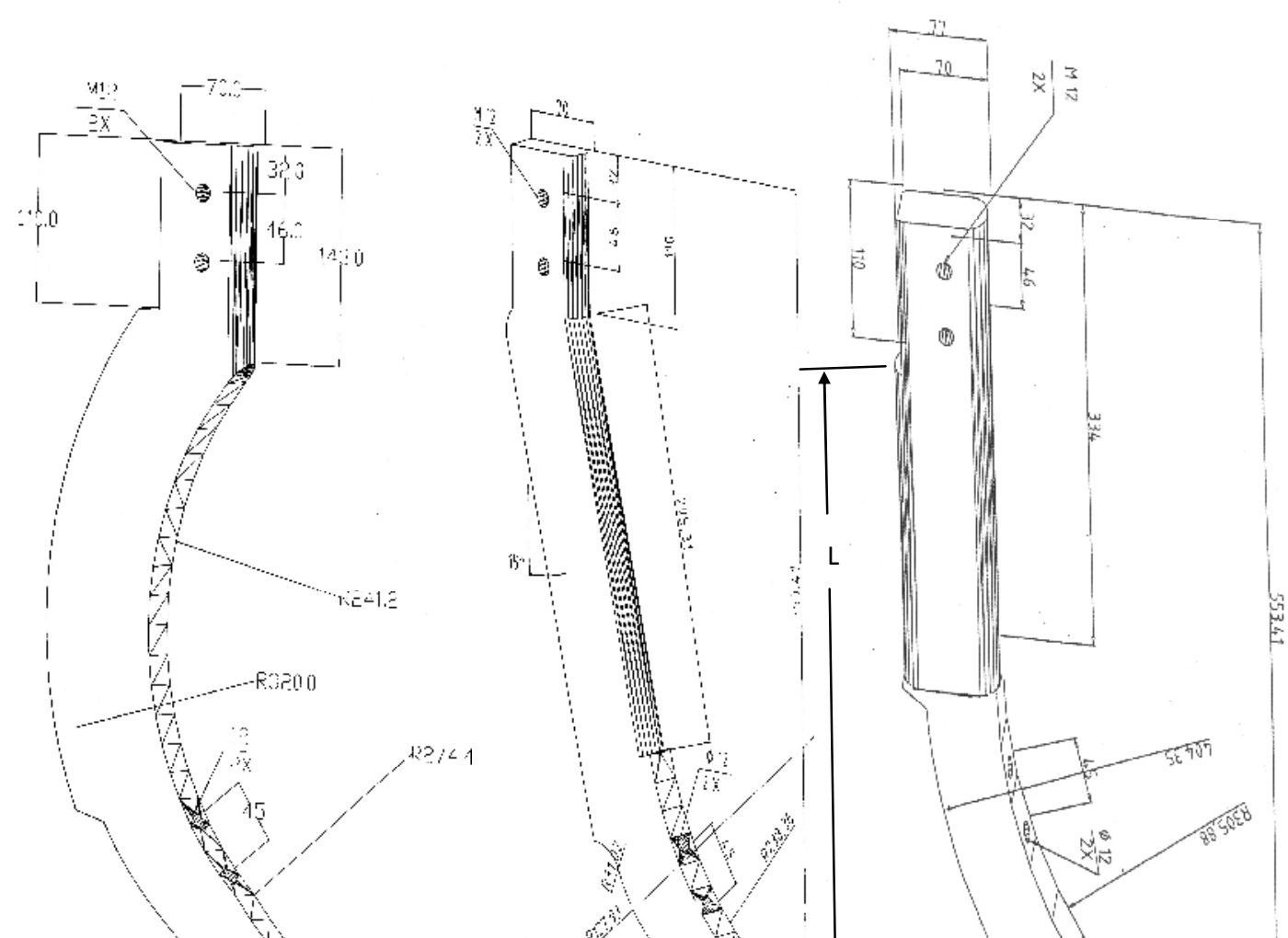


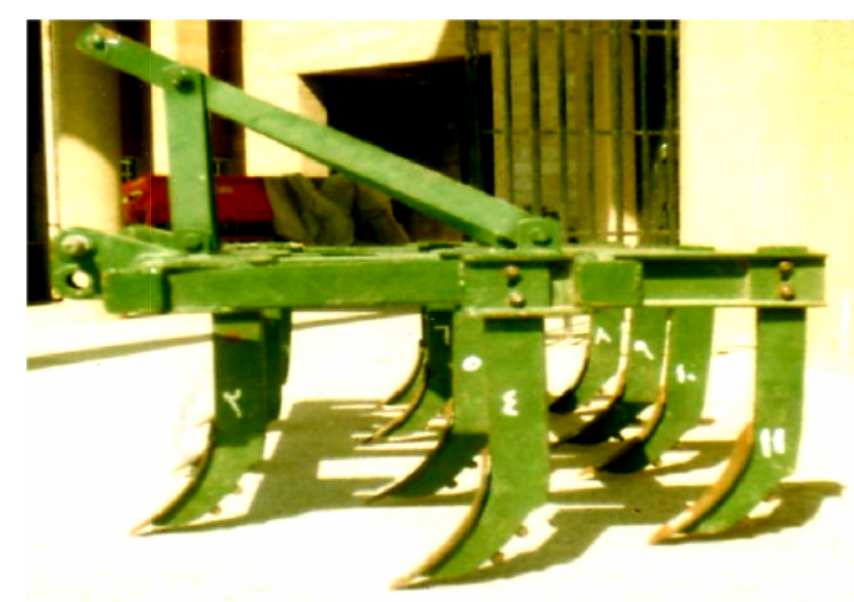



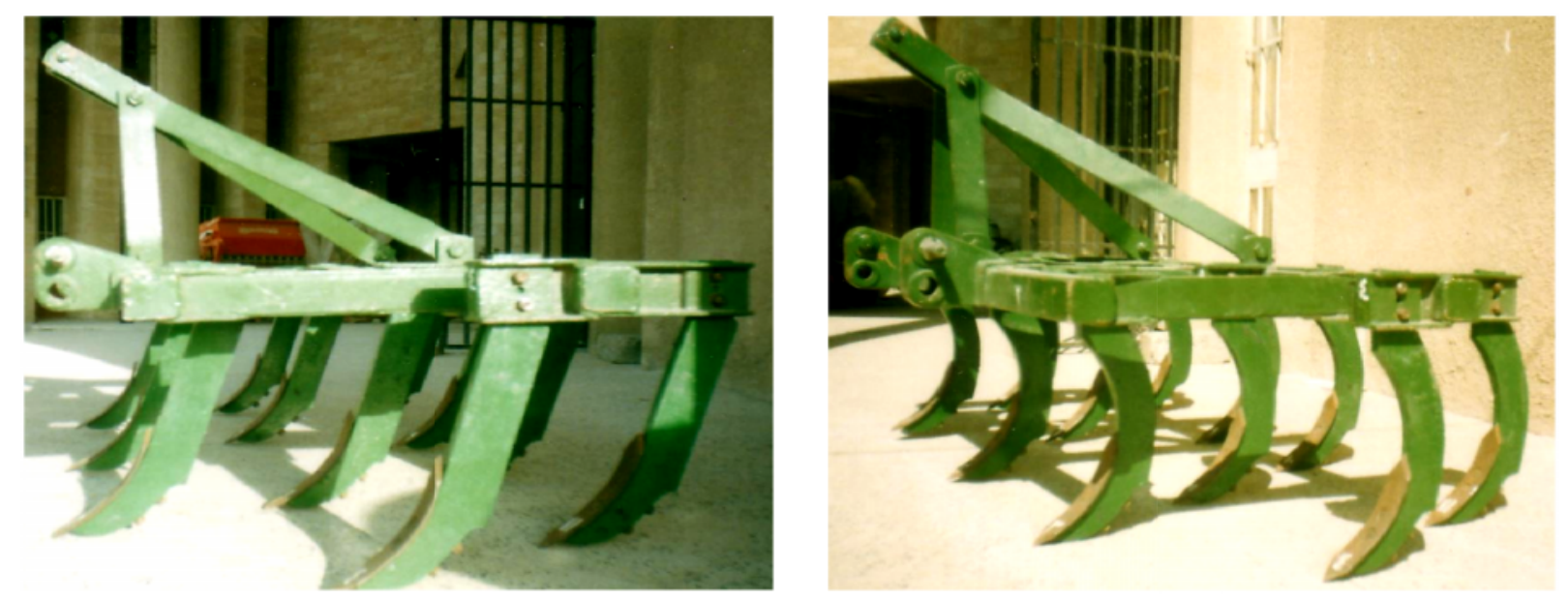

الشكل (3 ) : المحراث بالقصبات المنحرفة إلى الأمام.

الثكل (4 ) : المحراث بالقصبات المقوسة.

وتم حساب معدل

امـا معدل استهلاك الوقود فقد استخدم جهاز مصنع محليا أستهلاك الوقود حسب القانون التالي :

$$
\mathrm{Fc}=(\mathrm{Fc} 3-\mathrm{Fc} 2)+\mathrm{Fc} 1
$$

= استهلاك الوقود للساحبة قبد الدر اسة و الدحراث . Fc Fc1 استهلاك الوقود للساحبة الأمامية وحدها . Fc1 Fc2 = استهلاك الوقود للساحبتين و المحر اث معلق لا يعمل. 


$$
\text { Fc3 }
$$

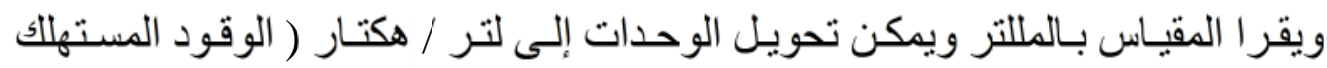

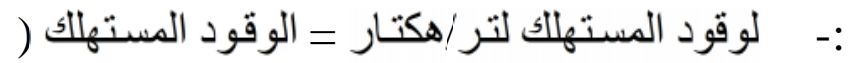

10000 x (

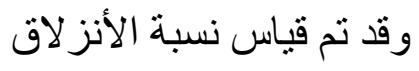

$$
\begin{array}{cl}
\text { Slip } \%=\{1-(\mathrm{Vp} / \mathrm{Vt})\} \times 100 & \text { حيث ان } \\
\mathrm{km} / \mathrm{h} & =\mathrm{Vp} \\
\mathrm{km} / \mathrm{h} & =\mathrm{Vt}
\end{array}
$$

حيث إن هذه الطريقة هي التي تم اعتمادها في حساب نسبة الانز لاق بعد أخذ الزمن

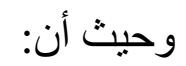

$$
\begin{aligned}
& \text { Vt = L / Tt السرعة النظرية } \\
& \text { Vp = L / Tp }
\end{aligned}
$$

m (

$\begin{aligned}) & =\mathrm{L} \\ \sec & =\mathrm{Tt} \\ \sec & =\mathrm{Tp}\end{aligned}$

= - الانز لاق بسبب سحب الساحبة الخلفية مع المحر اث أثناء

${ }^{[8]} \dot{\eta} \delta=\{\mathrm{Pk}(\mathrm{Vt}-\mathrm{Vp})\} 0.746 / 270$ : 


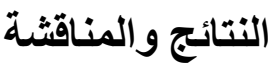

1 - تأثثر شكل قصبة المحر اث في الصفات المدروسة

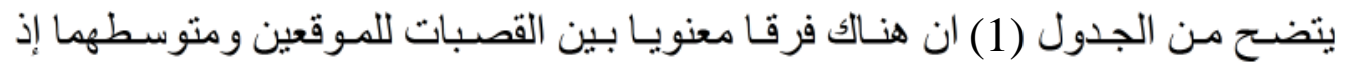

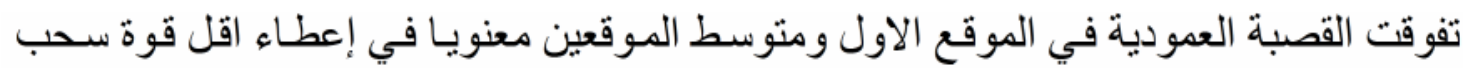

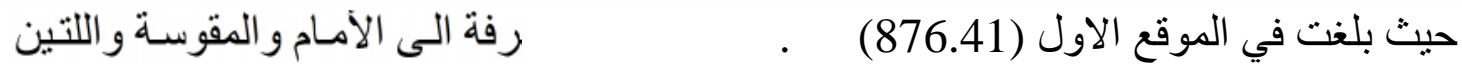

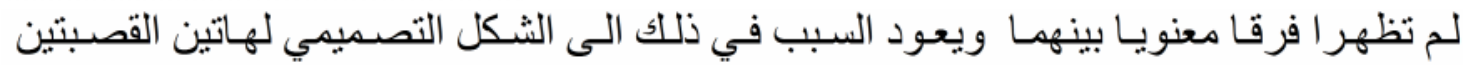

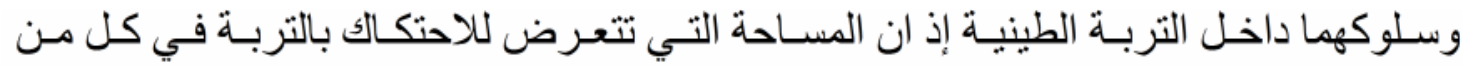

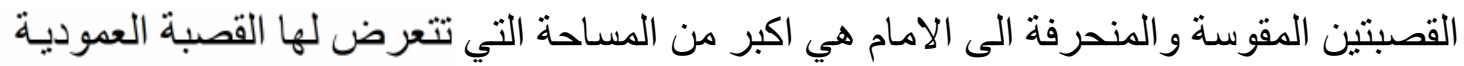

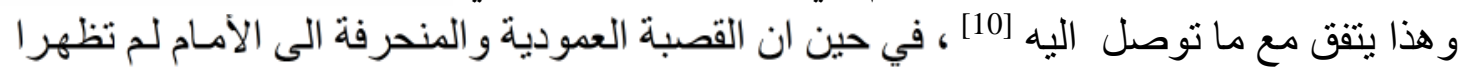

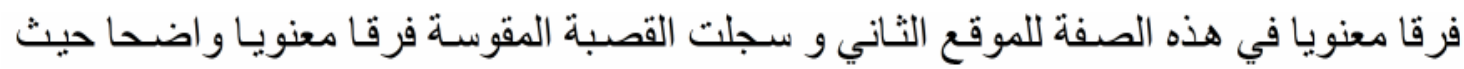

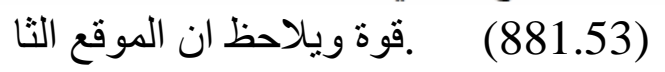

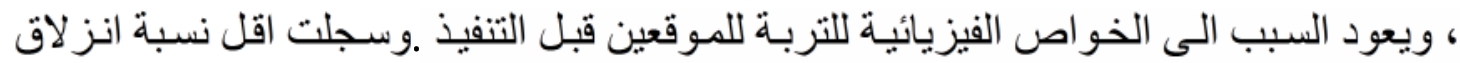

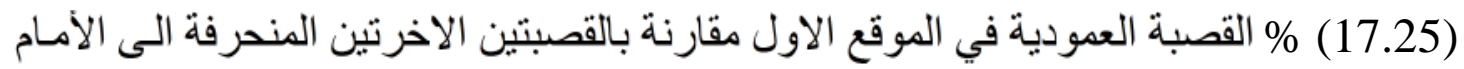

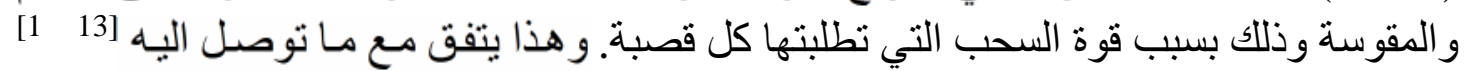

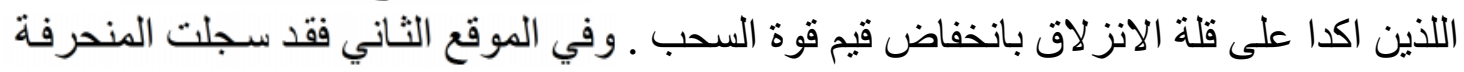

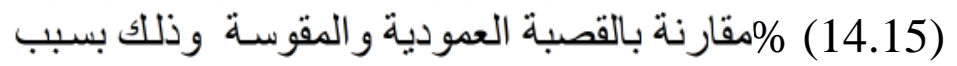

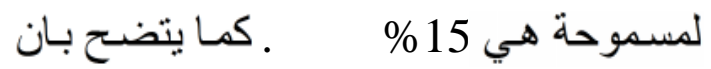

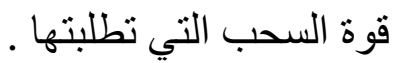

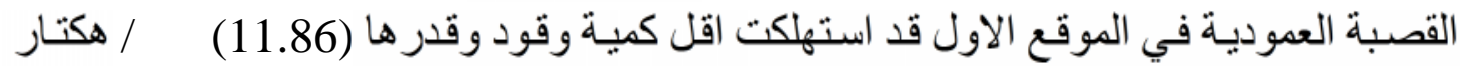

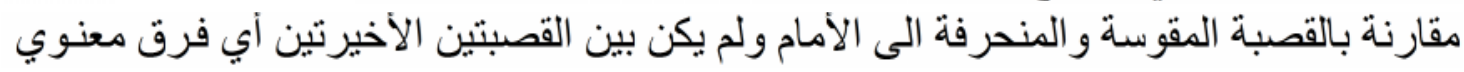

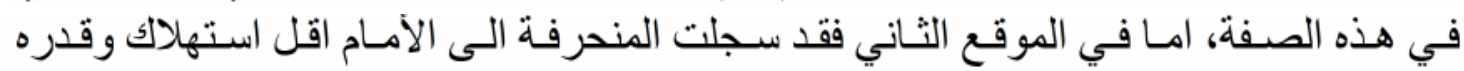

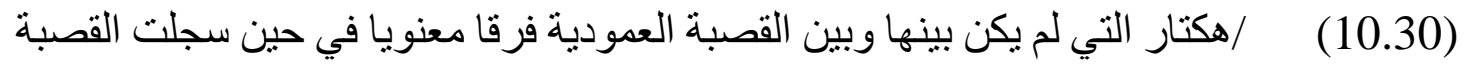

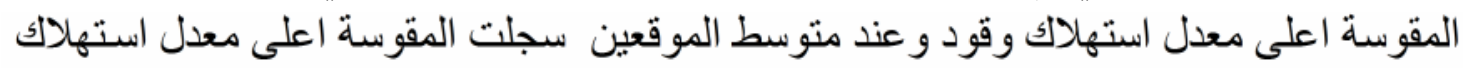

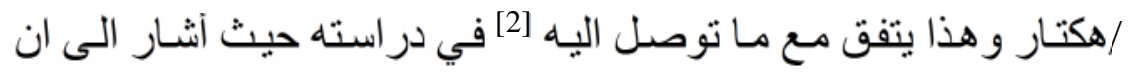

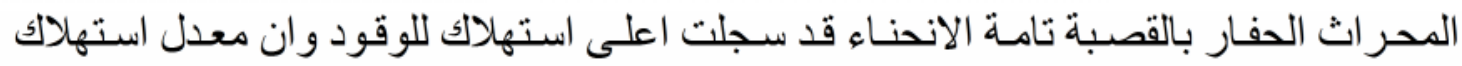

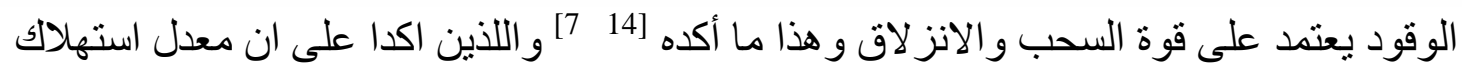

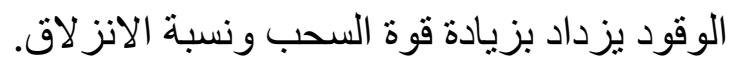

اما بالنسبة لصفتي قدرة السحب و القدرة المفقودة بالانز لاق فقد سجلتا نفس النتائج لانهما تعتمدان

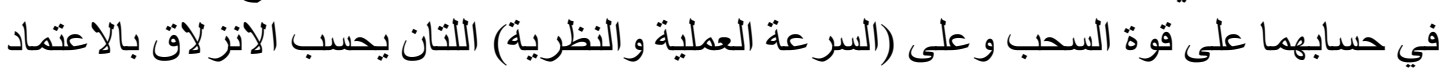

(1) يبين تأثير شكل القصبة في الصفات المدروسة

\begin{tabular}{|c|c|}
\hline الصفات المدروسة & شكل القصبة \\
\hline
\end{tabular}




\begin{tabular}{|c|c|c|c|c|c|c|}
\hline المفقودة & قدرة السحب & لتر الوكتود & الانزلاق & كفم.قوة & & \\
\hline $\begin{array}{c}4.21 \\
b\end{array}$ & $\begin{array}{c}18.39 \\
\mathrm{~b}\end{array}$ & $\begin{array}{c}11.86 \\
b\end{array}$ & $\begin{array}{c}17.25 \\
\mathrm{~b}\end{array}$ & $\begin{array}{c}876.41 \\
\text { b }\end{array}$ & العمودية & \multirow{3}{*}{$\begin{array}{l}\overline{3} \\
\text { :ू } \\
\overline{3}\end{array}$} \\
\hline $\begin{array}{c}5.51 \\
\mathrm{a}\end{array}$ & $\begin{array}{c}21.25 \\
\mathrm{a}\end{array}$ & 14.49 & 20.74 & 1072.24 & المنحرفة الى & \\
\hline $\begin{array}{c}\mathrm{a} \\
6.07 \\
\mathrm{a}\end{array}$ & $\begin{array}{c}22.06 \\
\mathrm{a}\end{array}$ & $\begin{array}{c}14.95 \\
\mathrm{a}\end{array}$ & $\begin{array}{c}21.20 \\
\mathrm{a}\end{array}$ & $\begin{array}{c}1101.41 \\
\mathrm{a}\end{array}$ & المقوسة & \\
\hline $\begin{array}{c}2.87 \\
b\end{array}$ & $\begin{array}{c}16.82 \\
\mathrm{~b}\end{array}$ & $\begin{array}{c}\mathrm{a} \\
10.42 \\
\mathrm{~b}\end{array}$ & $\begin{array}{c}14.34 \\
b\end{array}$ & $\begin{array}{c}740.31 \\
b\end{array}$ & العمودية & \multirow{3}{*}{ 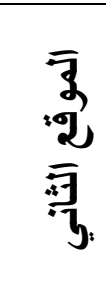 } \\
\hline $\begin{array}{c}2.59 \\
\mathrm{~b}\end{array}$ & $\begin{array}{c}16.11 \\
\mathrm{~b}\end{array}$ & $\begin{array}{c}10.30 \\
\mathrm{~b}\end{array}$ & $\begin{array}{c}14.15 \\
b\end{array}$ & $\begin{array}{c}731.98 \\
\text { b }\end{array}$ & المنحرفة الى & \\
\hline $\begin{array}{c}3.84 \\
\mathrm{a}\end{array}$ & $\begin{array}{c}18.46 \\
\mathrm{a}\end{array}$ & $\begin{array}{c}11.88 \\
\mathrm{a}\end{array}$ & $\begin{array}{c}17.06 \\
\mathrm{a}\end{array}$ & $\begin{array}{c}881.53 \\
\mathrm{a}\end{array}$ & المقوسة & \\
\hline $\begin{array}{c}3.54 \\
\mathrm{c}\end{array}$ & $\begin{array}{c}17.61 \\
\mathrm{c}\end{array}$ & $\begin{array}{c}11.14 \\
\mathrm{c}\end{array}$ & $\begin{array}{c}15.80 \\
\mathrm{c}\end{array}$ & $\begin{array}{c}808.36 \\
c\end{array}$ & العمودية & \multirow{3}{*}{ 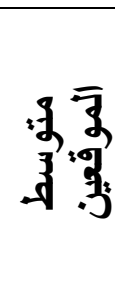 } \\
\hline $\begin{array}{l}4.05 \\
b\end{array}$ & $\begin{array}{c}18.68 \\
\text { b }\end{array}$ & $\begin{array}{c}12.40 \\
\mathrm{~b}\end{array}$ & $\begin{array}{c}17.44 \\
b\end{array}$ & $\begin{array}{c}902.11 \\
b\end{array}$ & المنحرفة الى & \\
\hline $\begin{array}{c}4.96 \\
\mathrm{a}\end{array}$ & $\begin{array}{c}20.26 \\
\mathrm{a}\end{array}$ & $\begin{array}{c}13.42 \\
\mathrm{a}\end{array}$ & $\begin{array}{c}19.13 \\
\mathrm{a}\end{array}$ & $\begin{array}{c}991.47 \\
\mathrm{a}\end{array}$ & المقوسة & \\
\hline
\end{tabular}

القيمة الأقل لكل صفة هي الأفضل

2- تأثثير عمق الحر اثة في الصفات المدروسة

يتضح من الجدول (2) وق العمق الاول في الموقعين ومتوسطهما على العمق الثاني

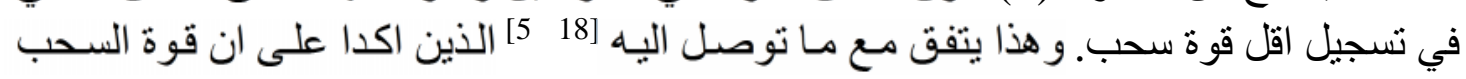
تزداد بزيادة عمق الحر اثة وذللك بسبب زيادة مقطع التربة المثارة من قبل الآلة.

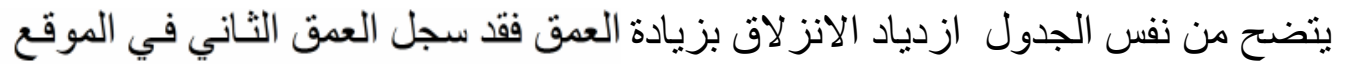

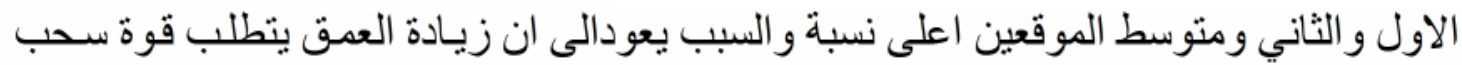

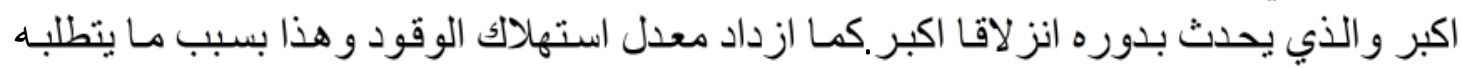

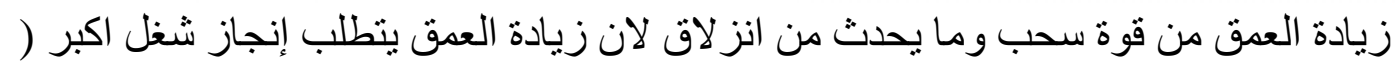

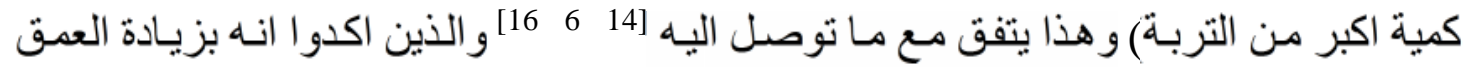

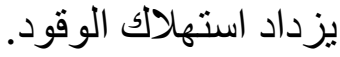

وفي صفة قدرة السحب لوحظو جود فرق معنوب ونوب بين الأعماق في هذه الصفة حيث سجل العمق الاول في الموقعين ومتوسطهي

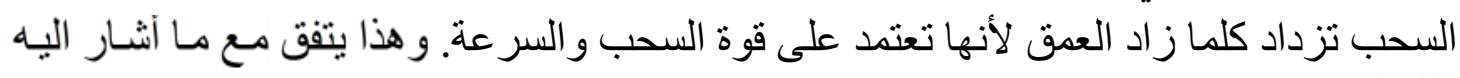


(2) يبين تأثير عمق الحر اثة في الصفات المدروسة

\begin{tabular}{|c|c|c|c|c|c|c|}
\hline $\mathrm{kW}$ & kW & $\begin{array}{l}\text { /هتهنار / هكتار } \\
\text { / }\end{array}$ & $\%$ & & & \\
\hline $\begin{array}{c}4.62 \\
b\end{array}$ & $\begin{array}{c}19.44 \\
\text { b }\end{array}$ & $\begin{array}{c}12.77 \\
b\end{array}$ & $\begin{array}{c}18.32 \\
\mathrm{~b}\end{array}$ & $\begin{array}{c}943.54 \\
\text { b }\end{array}$ & $(12-8)$ & \\
\hline $\begin{array}{c}5.90 \\
\mathrm{a}\end{array}$ & $\begin{array}{c}21.70 \\
\mathrm{a}\end{array}$ & $\begin{array}{c}14.77 \\
\mathrm{a}\end{array}$ & $\begin{array}{c}21.13 \\
\mathrm{a}\end{array}$ & \begin{tabular}{|c|}
1089.84 \\
$\mathrm{a}$
\end{tabular} & $(17-13)$ & \\
\hline $\begin{array}{c}2.70 \\
b\end{array}$ & $\begin{array}{c}16.22 \\
\mathrm{~b}\end{array}$ & $\begin{array}{c}10.06 \\
\mathrm{~b}\end{array}$ & $\begin{array}{c}13.99 \\
\text { b }\end{array}$ & $\begin{array}{c}723.81 \\
\mathrm{~b}\end{array}$ & $(12-8)$ & \\
\hline $\begin{array}{c}3.50 \\
\mathrm{a}\end{array}$ & $\begin{array}{c}18.04 \\
\mathrm{a}\end{array}$ & $\begin{array}{c}11.67 \\
\mathrm{a}\end{array}$ & $\begin{array}{c}16.37 \\
\mathrm{a}\end{array}$ & \begin{tabular}{|c|}
845.40 \\
$\mathrm{a}$
\end{tabular} & $(17-13)$ & \\
\hline $\begin{array}{c}3.66 \\
b\end{array}$ & $\begin{array}{c}17.83 \\
\mathrm{~b}\end{array}$ & $\begin{array}{c}11.41 \\
\mathrm{~b}\end{array}$ & $\begin{array}{c}16.15 \\
b\end{array}$ & $\begin{array}{c}833.67 \\
\text { b }\end{array}$ & $(12-8)$ & $\bar{s}$ \\
\hline $\begin{array}{c}4.70 \\
\mathrm{a}\end{array}$ & $\begin{array}{c}19.87 \\
\mathrm{a}\end{array}$ & $\begin{array}{c}13.22 \\
\mathrm{a}\end{array}$ & $\begin{array}{c}18.75 \\
a\end{array}$ & $\begin{array}{c}967.62 \\
a\end{array}$ & $(17-13)$ & \\
\hline
\end{tabular}

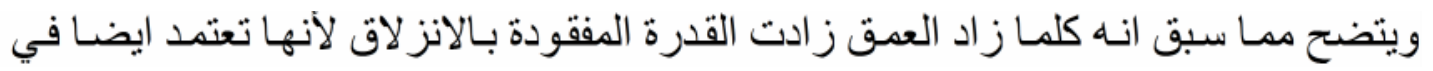

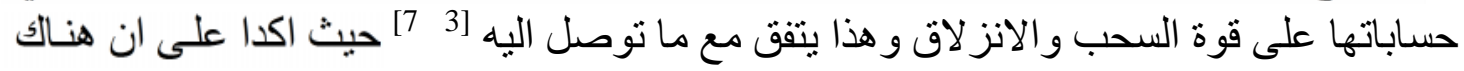
علاقة طرديه بين العمق و القدرة الدفقودة بالانز لاق ولاق

3- تأثير السرعة الأرضية في الصفات المدروسة

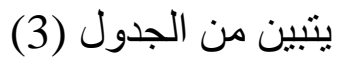

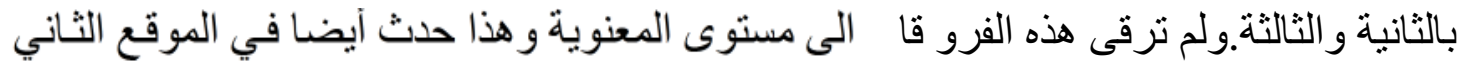

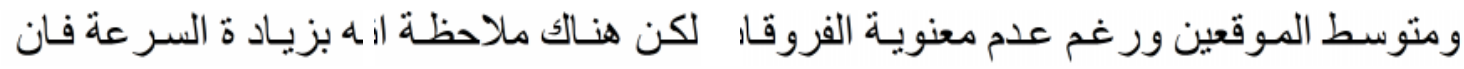

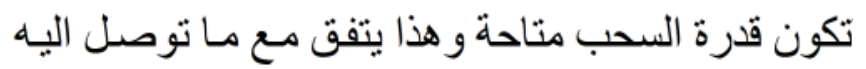

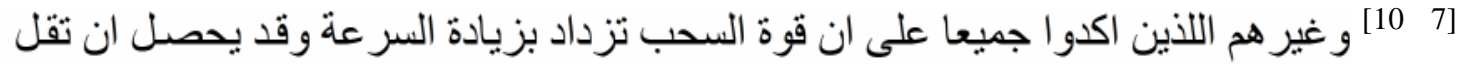

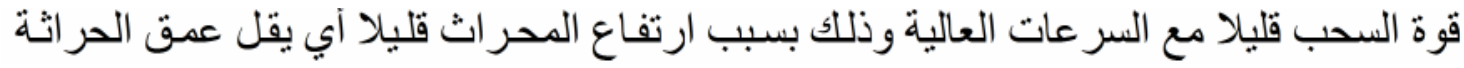

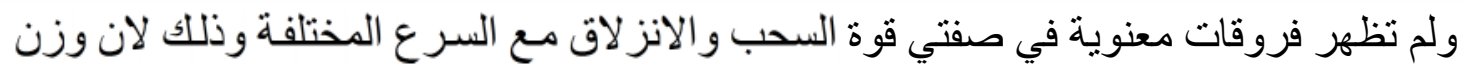

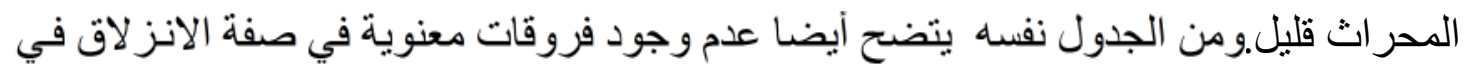

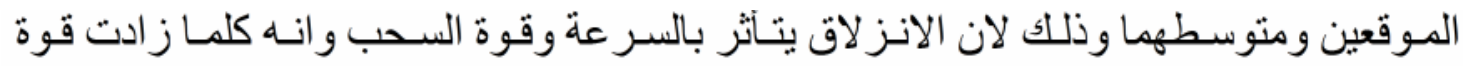

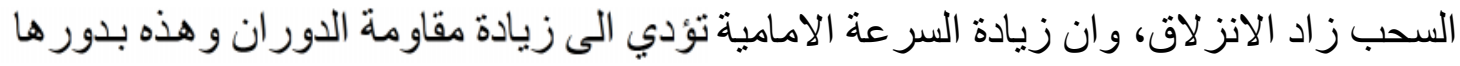

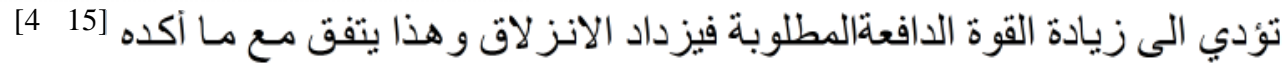


استهلاك الوقود لتر / هكتار سجلت السر عة الثالثة اقل استهلاك للوقود ومن ثم السر عة الثانية

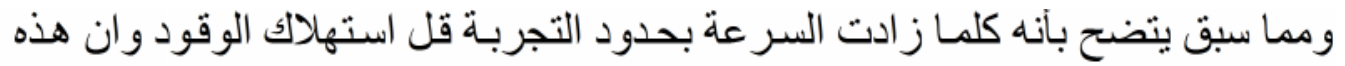

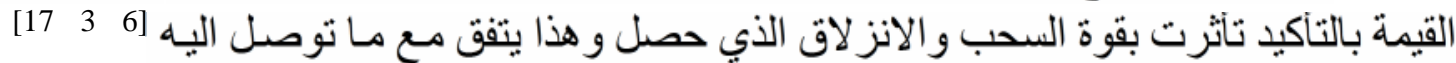

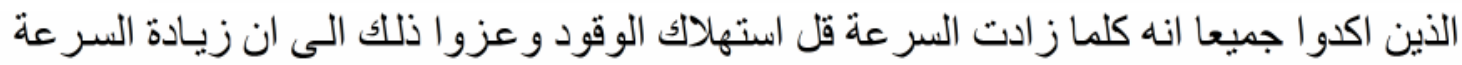
حبة المتاحة بشكل افضل أي زيادة انتاجية الساحبة .

فيتضـح من الجدول انه بالموقع الاول والثاني ومتوسط الموقعين kW

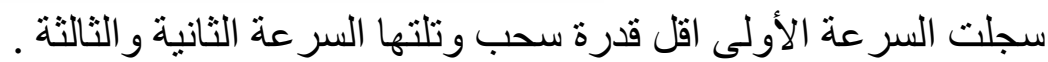

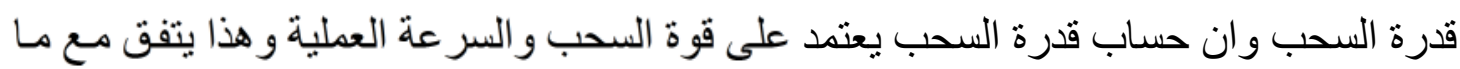

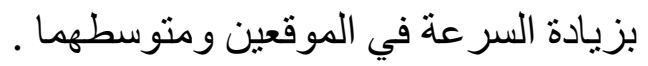
توصل اليه [9] وازدادت

(3) يبين تأثير السر عة الأرضية في الصفات الدروسة

\begin{tabular}{|c|c|c|c|c|c|c|}
\hline $\mathrm{kW}$ & $\mathrm{kW}$ & / استهلاك & $\%$ & - & الأرضية & \\
\hline $\begin{array}{c}2.75 \\
\mathrm{c}\end{array}$ & $\begin{array}{c}10.92 \\
\mathrm{c}\end{array}$ & $\begin{array}{c}18.21 \\
\mathrm{a}\end{array}$ & 19.49 & 1002.78 & 3.70 & \\
\hline $\begin{array}{c}5.62 \\
\mathrm{~b}\end{array}$ & $\begin{array}{c}21.59 \\
\text { b }\end{array}$ & $\begin{array}{c}13.09 \\
\text { b }\end{array}$ & 19.84 & 1022.22 & 7.15 & \\
\hline $\begin{array}{c}7.42 \\
\mathrm{a}\end{array}$ & $\begin{array}{c}29.20 \\
\mathrm{a}\end{array}$ & $\begin{array}{c}10.01 \\
\mathrm{c}\end{array}$ & 19.86 & 1025.07 & 9.64 & \\
\hline $\begin{array}{c}1.56 \\
\mathrm{c}\end{array}$ & $\begin{array}{c}8.74 \\
\mathrm{c}\end{array}$ & $\begin{array}{c}13.21 \\
\mathrm{a}\end{array}$ & 14.56 & 755.18 & 3.70 & \\
\hline $\begin{array}{c}3.48 \\
b\end{array}$ & $\begin{array}{c}18.51 \\
\text { b }\end{array}$ & $\begin{array}{c}11.53 \\
\mathrm{~b}\end{array}$ & 15.80 & 816.70 & 7.15 & \\
\hline $\begin{array}{c}4.27 \\
\mathrm{a}\end{array}$ & $\begin{array}{c}24.14 \\
\mathrm{a}\end{array}$ & $\begin{array}{c}7.86 \\
\mathrm{c}\end{array}$ & 15.19 & 781.94 & 9.64 & \\
\hline $\begin{array}{c}2.16 \\
\mathrm{c}\end{array}$ & $\begin{array}{c}9.83 \\
\mathrm{c}\end{array}$ & $\begin{array}{c}15.71 \\
\mathrm{a}\end{array}$ & 17.03 & 878.98 & 3.70 & : \\
\hline $\begin{array}{c}4.55 \\
\mathrm{~b}\end{array}$ & $\begin{array}{c}20.05 \\
\text { b }\end{array}$ & $\begin{array}{c}12.31 \\
\mathrm{~b}\end{array}$ & 17.82 & 919.46 & 7.15 & $\frac{1}{3}$ \\
\hline $\begin{array}{c}5.84 \\
\mathrm{a}\end{array}$ & $\begin{array}{c}26.67 \\
\mathrm{a}\end{array}$ & $\begin{array}{c}8.94 \\
\mathrm{c}\end{array}$ & 17.52 & 903.51 & 9.64 & ? \\
\hline
\end{tabular}

القيمة الأقل لكل صفة هي الأفضل. 
(4) يتضح بان هنالك فرق معنوي بين الموقعين في كل متطلبات القدرة فقد سجل

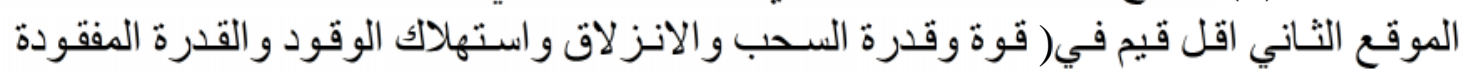

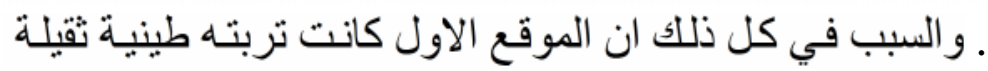

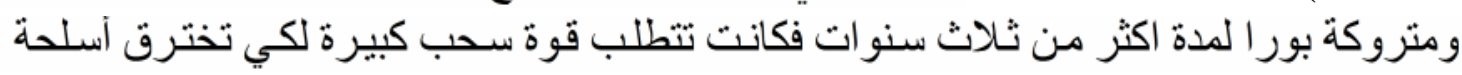

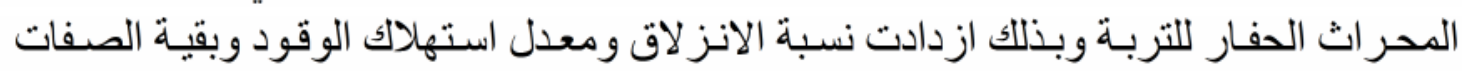

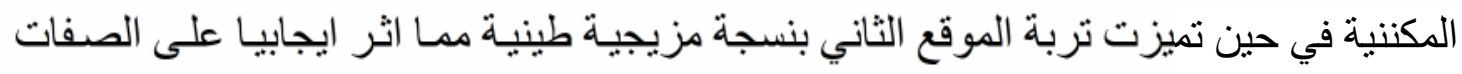

(4) تأثثر المو اقع في الصفات المدروسة

\begin{tabular}{|c|c|c|c|c|}
\hline $\mathrm{kW}$ & $\mathrm{kW}$ & $\begin{array}{l}\text { /هتهنار / هكتار } \\
\text { / }\end{array}$ & $\%$ & * \\
\hline $5.26 \mathrm{a}$ & $20.57 \mathrm{a}$ & $13.77 \mathrm{a}$ & $19.73 \mathrm{a}$ & $1016.69 a$ \\
\hline $3.10 \mathrm{~b}$ & $17.13 b$ & $10.87 \mathrm{~b}$ & $15.18 \mathrm{~b}$ & $784.61 b$ \\
\hline
\end{tabular}

5ـ تأثير التاخل بين المواقع وشكل القصبة والعمق والسرعة في الصفات المدروسة

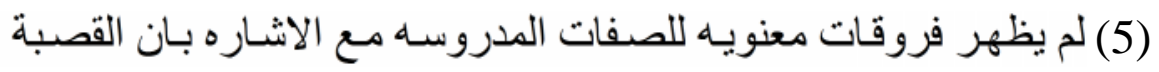

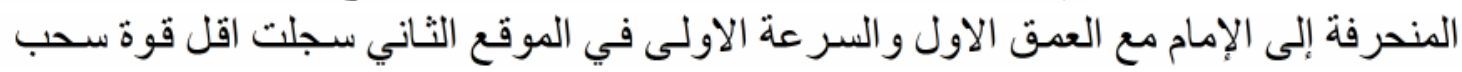
kW (7.22) \% (11.23)

kW (0.91)

الثناني اقل استهلاك للوقود وقدره (6.75) / هكتار.

(5 ) نأثثر التداخل بين المواقع وشكل القصبة والعمق و السرعة في الصفات المدروسة

\begin{tabular}{|c|c|c|c|c|c|c|c|c|}
\hline \multicolumn{5}{|c|}{ الصفات المدروسة } & \multirow{2}{*}{ 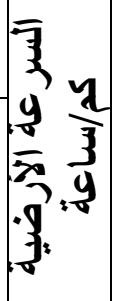 } & \multirow{2}{*}{$\begin{array}{l}\bar{y} \\
\bar{y} \\
\bar{y} \\
\overline{3} \\
\overline{3} \\
\bar{x}\end{array}$} & \multirow{2}{*}{ 羿 } & \multirow[b]{2}{*}{$\frac{\overline{3}}{\text { बे }}$} \\
\hline بالانزلاقدة & السحب & | الوقود لتر / معلد / & الانزلاق \% & كفرة السحب & & & & \\
\hline
\end{tabular}




\begin{tabular}{|c|c|c|c|c|c|c|c|c|}
\hline 1.62 & 8.88 & 13.96 & 14.95 & 766.67 & 3.70 & & \multirow{6}{*}{$\begin{array}{l}\overline{3} \\
\overline{3} \\
\overline{3}\end{array}$} & \\
\hline 1.81 & 9.33 & 15.36 & 16.67 & 841.67 & 7.15 & $\stackrel{1}{1}$ & & \multirow{16}{*}{$\begin{array}{l}\overline{3} \\
\text { :ू }\end{array}$} \\
\hline 4.49 & 17.49 & 10.32 & 16.41 & 791.67 & 64.9 & & & \\
\hline 5.83 & 22.47 & 13.99 & 20.83 & 1075.00 & $\begin{array}{r}.3 \\
70\end{array}$ & \multirow{3}{*}{$\stackrel{\vec{\omega}}{\stackrel{\nu}{\nu}}$} & & \\
\hline 3.66 & 22.23 & 7.13 & 14.04 & 725.07 & $\begin{array}{l}.7 \\
15 \\
\end{array}$ & & & \\
\hline 7.84 & 29.95 & 10.40 & 20.61 & 1058.40 & $\begin{array}{l}9 \\
64\end{array}$ & & & \\
\hline 2.66 & 11.04 & 18.11 & 19.37 & 1000.00 & 70.3 & \multirow{6}{*}{ دَ } & & \\
\hline 3.98 & 12.69 & 21.89 & 23.60 & 1216.67 & 15.7 & & 3 & \\
\hline 5.67 & 22.35 & 13.05 & 20.19 & 1058.33 & 9.64 & & & \\
\hline 6.53 & 23.26 & 13.95 & 21.82 & 1125.00 & $\begin{array}{c}.3 \\
70\end{array}$ & & $\overline{3}$ & \\
\hline 6.65 & 28.55 & 9.73 & 19.22 & 991.73 & 15.7 & & 3 & \\
\hline 7.57 & 29.62 & 10.23 & 20.23 & 1041.73 & 64.9 & & & \\
\hline 2.91 & 11.36 & 18.97 & 20.00 & 1041.67 & 70.3 & \multirow{3}{*}{ 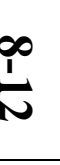 } & \multirow{6}{*}{-3. } & \\
\hline 3.54 & 12.22 & 20.96 & 22.34 & 1150.00 & 15.7 & & & \\
\hline 4.69 & 20.69 & 12.50 & 18.32 & 958.33 & 64.9 & & & \\
\hline 4.49 & 23.29 & 14.72 & 21.46 & 1125.00 & 70.3 & - & & \\
\hline 9.01 & 32.07 & 10.95 & 22.06 & 1141.73 & 15.7 & I & & \\
\hline 9.78 & 32.76 & 11.63 & 22.99 & 1191.73 & 64.9 & $\checkmark$ & & \\
\hline 0.96 & 7.47 & 11.25 & 11.86 & 616.73 & 3.70 & \multirow{3}{*}{$\frac{\sigma}{\infty}$} & \multirow{6}{*}{ 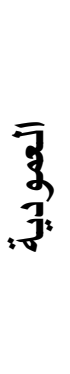 } & \multirow{18}{*}{ 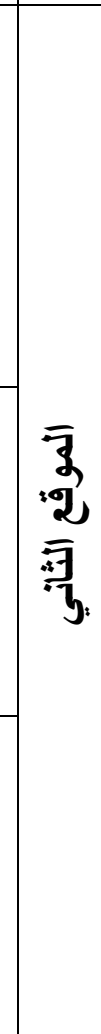 } \\
\hline 1.40 & 8.23 & 13.62 & 14.01 & 725.07 & 7.15 & & & \\
\hline 2.65 & 17.59 & 10.34 & 13.89 & 716.70 & 9.64 & & & \\
\hline 3.06 & 17.98 & 11.13 & 15.28 & 783.37 & 3.70 & & & \\
\hline 4.26 & 23.93 & 7.63 & 15.07 & 775.001 & $\begin{array}{l}7 . \\
15\end{array}$ & $\frac{5}{g}$ & & \\
\hline 4.86 & 25.74 & 8.56 & 15.95 & 825.00 & 9.64 & & & \\
\hline 0.91 & 7.22 & 10.86 & 11.23 & 591.73 & 3.70 & \multirow{3}{*}{$\infty$} & \multirow{6}{*}{ 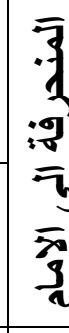 } & \\
\hline 1.67 & 9.21 & 14.86 & 15.36 & 791.73 & 7.15 & & & \\
\hline 3.13 & 17.54 & 10.66 & 15.00 & 775.03 & 9.64 & & & \\
\hline 3.23 & 17.88 & 11.06 & 15.28 & 791.70 & 3.70 & $n$ & & \\
\hline 2.44 & 20.17 & 6.75 & 12.32 & 633.33 & 7.15 & $\bar{n}$ & & \\
\hline 4.18 & 24.67 & 8.03 & 15.71 & 808.33 & 9.64 & - & & \\
\hline 1.50 & 8.92 & 13.06 & 14.81 & 764.07 & 3.70 & & \multirow{6}{*}{ 恿 } & \\
\hline 2.90 & 11.41 & 16.03 & 20.12 & 1041.73 & 7.15 & & & \\
\hline 3.79 & 18.88 & 12.33 & 16.39 & 850.03 & 9.64 & & & \\
\hline 5.00 & 21.23 & 13.66 & 18.98 & 983.37 & 3.70 & \multirow{3}{*}{$\bar{n}$} & & \\
\hline 4.65 & 24.33 & 7.67 & 15.41 & 791.37 & 7.15 & & & \\
\hline 5.22 & 26.00 & 8.53 & 16.67 & 858.33 & 9.64 & & & \\
\hline
\end{tabular}


1-افضل القصبات استخداما في الترب الطينية هي القصبة العمودية في حين ان القصبة الم

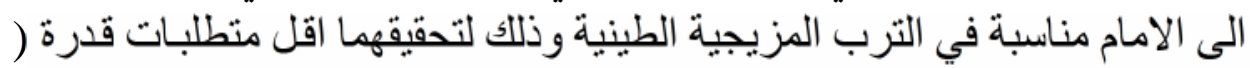
السحب،استهلاك الوقود،نسبة الانز لاق، القدرة المفقودة بالانز لاق).

$$
\text { 2-زيادة عمق الحر اثة زاد من متطلبات القدرة. }
$$

3-زيادة السر عة الارضيفتزيد من متطلبات القدرة غالبـا ماعدا استهلاك الوقود حيث يقل تبعا

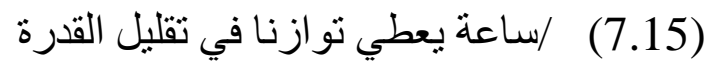

4-تفوق الموقع الثاني في معظم الصفات المكننية بالمقارنة مع الموقع الاول

1. البنـا، عزيـز رمـو معدات تهيئة التربـة، مديريـة دار الكتب للطباعـة و النشـر، جامعـة

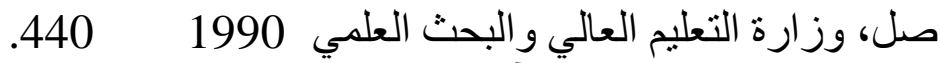
ـ تأثنير شكل قصبة المحر اث الحفار على الإنتاجية وطاقة

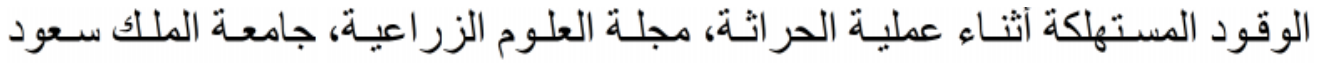

$$
\text { . 2-1 } 200517 \text { (1) }
$$

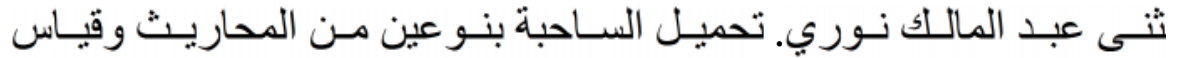

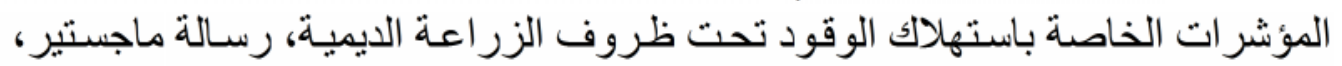

قسم المكننة الزر اعية، كلية الزر اعة والغابات، جامعة الموصل 1998 المولت 190

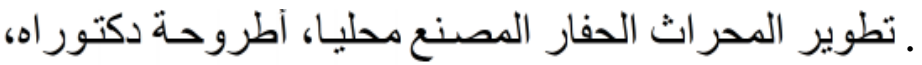

قسم المكننة الزر اعية، كلية الزر اعة و الغابات، جامعة الموصل $2005 \quad 225$. 


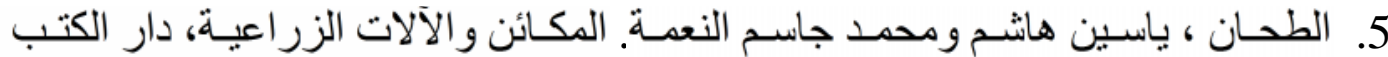

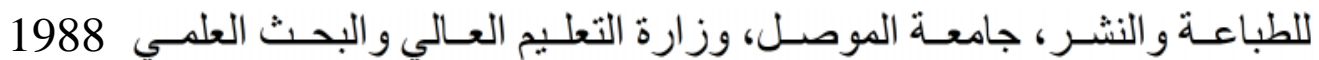

.419

ياسين هاشم. تأثير المحتوى الرطوبي باستخدام أنواع مختلفة من المحاريث

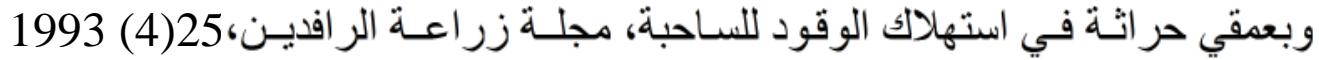

$.49-45$

7. المشرقي ، سمير عبد الله علي. تطوير اذرع الثبك وتاثير هـا في اداء السـاحبة المحملة

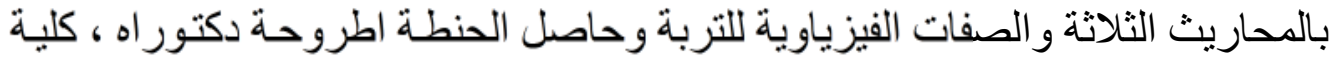
303 1999

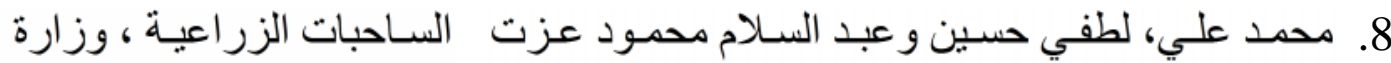

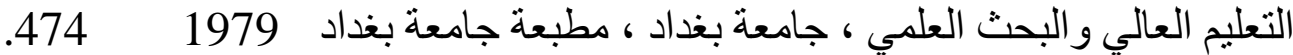

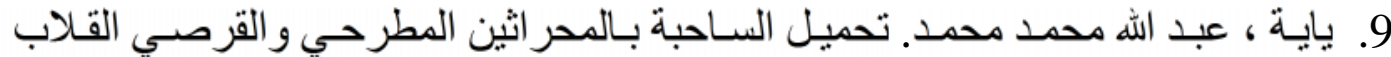

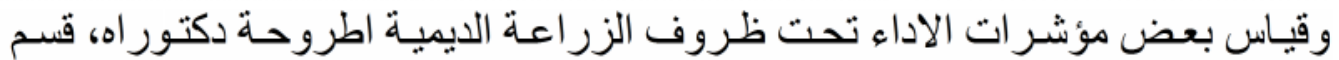

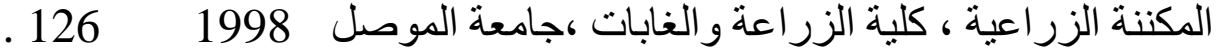

10.Aljanobi, A. A. and Suhaibani, S. A.; Draft of primary Tillage implements in Sandy Loam Soil transaction of ASAE, 14.(4), 1998: 343-348.

11.Aljanobi, A.A.; Wahby, M.F., Abu Karima, A.M. and Al-hammed, S.A.Influence of chisel plow shank shapes on Horizontal and Vertical force Requirements. Agri. Sci.J, 7 (1), 2002 : 13-19.

12.Baloch, J.M.; S.B. Bukhari, S.N. Mirani and A.N Mirani, Power Requirements of Tillage Implements. Agricultural Mechanization in Asia, Africa and Latin America 22(1), 1991, 34-38.

13.Bukhari, S.;M.A. Bhutto; J.M.Baloch; A.B.Bhutto and A.N. Miran.. Performance of . selected tillage implements . Agri. Mech. In Asia, Africa and Latin America. 19 (4), 1988 : 9-14.

14.Frisby, J. C. And Summers, J. D.; Energy - related data for selected implements. Trans. Of ASAE, 22(5), 1979: 1010-1011.

15.Gunderson, D. G.; Kirk, T. G.; Wilson, J, N. And Dyck, F. B.; Draftspeed-depth characteristics of cultivators and discers and their effect on fuel consumption. Trans. Of ASAE, 81(1), 1981: 16031612.

16.Hula, J.; koraricek, P.; Mayer, V.; Podpera, V. And Vlaskova, M.; Chosen machine in technologies of Soil protective Tillage and their energy consumption. Research in stitute of Agricultural Engineering, 6-Ruzyne, Czech Republic, 2002 : 44-49.

praque

17.Onwualu, A. P. And Watts, K. C.; Draught and vertical forces obtained from dynamic Soil cutting by plane Tillage tools. Soil and Tillage Res, 48(4), 1998: 239-253.

18. Willcocks, T. J.; Tillage of clode-forming Sand Loam Soil in the Semi - Arid climate of bot swana. Soil and Tillage Res., 1(1), 1981: 325350 . 
